\title{
Magnetic Interference Analysis Caused by Magnetic Survey Pod Track Fluctuation in Helicopters
}

\author{
Yuhui Sun ${ }^{1,2, a}$, Chunsheng Lin ${ }^{1, b}$, Jianyang Zhao ${ }^{1, c}$,and Guojun Zhai ${ }^{3, d}$ \\ ${ }^{1}$ Department of Weapon Engineering, Naval University of Engineering, Wuhan 430033, China; \\ ${ }^{2}$ New Star Research Institute of Applied Tecnology, Hefei 230031, China; \\ ${ }^{3}$ Naval Institute of Hydrographic Surveying and Charting Tianjin 300061, China. \\ a sunyh389@163.com, ${ }^{b}$ Ics_and_zh@163.com, 'zhao1986213 @126.com, 'zhaigj@163.com
}

Keywords: magnetic survey pod; latitude fluctuation; altitude fluctuation; magnetic interference.

\begin{abstract}
The track fluctuation of helicopter pod used for magnetic survey can cause interference with magnetic field measurement results. By simulating the small fluctuation of inclination angle and drift angle, the pod track fluctuation in airborne coordinate system and the helicopter track fluctuation in geodetic coordinate system are analyzed in detail, and the track fluctuation model is established and calculated in a simulated environment. Results indicate the magnetic interference rules of the track fluctuation, and can provide a theoretical support for magnetic interference compensation.
\end{abstract}

\section{Introduction}

At present, the aeromagnetic survey technology has been widely used in civil and military fields. In civilian areas, it is mainly used in resource exploration, geological survey and geomagnetic navigation applications, and in military areas mainly in anti-submarine with airborne magnetic anomaly and geomagnetic navigation. When magnetic anomaly detection used in helicopter platform, it has advantages of high efficiency, passive detection respect to the acoustic detection means, and compared to other sports platform, it has advantages of high precision, and less effect of taking off and landing conditions, and so on.

Relative to the earth coordinate system, as the effect of aircraft's maneuvering and crosswind, the pod latitude fluctuation and altitude fluctuation occurs, and inevitably leads to the magnetic interference while measuring. This interference is an important part of the helicopter platform background magnetic interference. To measure the magnetic anomaly in high precision, it must first analyze and compensate the various background magnetic interferences. Liu et al. ${ }^{2}$ and Zhou et al. ${ }^{3}$ respectively analyzed the background magnetic interferences of fixed-wing aircraft and unmanned aerial vehicle (UAV), established the relevant models and compensate the interferences. But these methods are not completely suitable for helicopter magnetic pod. Therefore the main work of this paper is to analyze track fluctuation of the helicopter magnetic pod, and model the magnetic interference, which can provide theoretical basis for the subsequent background magnetic interference compensation.

\section{Track fluctuation model}

Pod track fluctuation is consisting of two parts: pod track fluctuations in airborne coordinate system and the aircraft track fluctuation in geodetic coordinate system.

\subsection{Pod track fluctuations in airborne coordinate system}

In Fig.1, OXYZ is the airborne coordinate system. Regard the suspension point $O_{1}$ as the origin, the geomagnetic coordinate system $O_{1} X_{1} Y_{1} Z_{1}$ is established. 


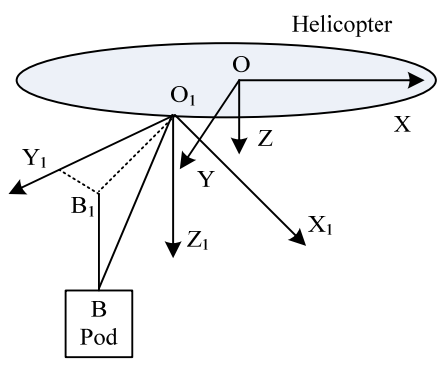

Fig. 1 coordinate systems schematic

The helicopter is assumed to fly in straight line, and $\theta_{0}$ is the average magnetic heading. As the wind resistance of pod rope is small, the rope $O_{1} B$ can be approximately equivalent to a straight line with length $L$.Pod inclination $I$ is the angle between rope and the horizontal plane, and pod deflection is the angle between the magnetic north and the projection in the horizontal plane of the rope.

If the helicopter flies ideal smoothly, there will be $I=70^{\circ}$ and $\theta=\theta_{0}$. And when the flight has slight fluctuation, it can be read as that the inclination and deflection are a constant value with an addition of a small variable. The magnetic declination of measurement point is $\theta_{d}$, the coordinates of $O_{1}$ in airborne coordinate system are $(a, b, c)$, and $(\theta, I)$ can be measured directly. So the coordinates of the pod in airborne coordinate system can be written as

$$
\left\{\begin{array}{l}
x=L \cdot \cos I \cdot \cos \left(\theta+\theta_{d}-\theta_{0}\right)+a \\
y=L \cdot \cos I \cdot \sin \left(\theta+\theta_{d}-\theta_{0}\right)+b \\
z=L \cdot \sin I+c
\end{array}\right.
$$

Taking inclination for example, we can simulate the fluctuations by models. Assuming the three kinds of center maneuvering frequencies of the helicopter as $f_{0}=0.1 H_{z}$, include pitching, rolling and yawing, frequency sequence $f_{i}=i^{*} f_{0} / 10, i=1,2 \ldots, N, N=20$, can be obtained.

The small change of inclination can be expressed as a combination of changes $\left(\Delta I_{1}, \Delta I_{2} \mathrm{~L}, \Delta I_{N}\right)$ in each frequency. According to the realistic environment, we set the max inclination $\Delta I_{m 1}=5^{\circ}$ when $f_{0}=0.1 H_{z}$. Then the small change $\Delta I_{i}=\Delta I_{m} \cdot F_{i}$, where $F_{i}=\exp \left(-4\left(f_{i}-f_{0}\right)^{2} / f_{0}^{2}\right)$, $\Delta I_{m}=\Delta I_{m 1} / G_{i} \approx 0.401 \Delta I_{m 1}$, and $G_{i}=\sqrt{\sum_{i=1}^{N} F_{i}}$.

Then model of the small change of inclination can be written as follows:

$$
\Delta I(t)=\sum_{i=1}^{N} \Delta I_{i} \sin \left(2 \pi f_{i} t+\varphi_{1 i}\right)+e_{I}(t)
$$

where $\varphi_{1 i}$ is a random phase distributed evenly in $(0,2 \pi), e_{I}(t)$ is a random noise obeying the normal distribution $N\left(0, \sigma_{1}^{2}\right), \sigma_{1}^{2}=\Delta I_{m}^{2} / 2 K^{2}$, and $K$ is signal-noise ratio.

\subsection{Latitude fluctuation model}

Latitude fluctuation of pod track in geodetic coordinate system is the north offset of pod displacement, including the offset of flight track and of the pod relative to the aircraft caused by pod swing. It can be showed as $x_{l}=x_{n l}+x_{h l}$.

(1) North offset of pod displacement

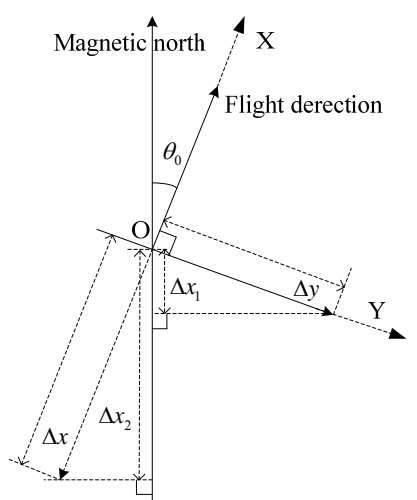

Fig.2 North offset of pod displacement 
As the wind and sling tension, the pod swings around. In Fig.2, $\Delta x$ and $\Delta y$ can be obtained by doing subtraction between Eq.1 and Eq.1 in addition of small fluctuation, then the north offset of pod displacement can be derived as follows:

$$
x_{n l}=-\Delta x \sin \theta_{0}-\Delta y \sin \theta_{0}=-(\Delta x+\Delta y) \sin \theta_{0}
$$

(2) North offset of flight track

$V_{0}$ is the flight velocity, $\theta_{0}$ is the average magnetic heading, and $\theta_{h}$ is the yaw angle, then the north velocity $V_{N}$ can be written as follows:

$$
V_{N}=V_{0} \cos \left(\theta_{0}+\theta_{h}\right)=V_{0} \cos \theta_{0} \cos \theta_{h}-V_{0} \sin \theta_{0} \sin \theta_{h} \approx V_{0} \cos \theta_{0}-V_{0} \theta_{h} \sin \theta_{0}
$$

$\theta_{h}$ can be obtained from the model of the small change of inclination, and then the northward component of the flight velocity and the northward displacement at $k$ point can be written as follows:

$$
\begin{gathered}
V_{N}(k)=V_{0} \cos \theta_{0}-V_{0} \sin \theta_{0} \cdot \theta_{h}(k) \\
X_{h l}(k)=V_{0} \cdot \cos \theta_{0} \cdot\left(t_{k}-t_{0}\right)-\sum_{j=1}^{k} V_{0} \sin \theta_{0} \cdot \theta_{h}(j) \Delta t
\end{gathered}
$$

where $\Delta t=t_{k}-t_{k-1}$ is sampling interval.

The first item of Eq.(6) is linear function, can be removed by filter, so the offset of northward displacement can be written as follows:

\subsection{Altitude fluctuation model}

$$
x_{h l}(k)=-\sum_{j=1}^{k} V_{0} \sin \theta_{0} \cdot \theta_{h}(j) \Delta t
$$

Altitude fluctuation of pod track in geodetic coordinate system includes the altitude fluctuation of flight track and of the pod relative to the aircraft caused by pod swing. It can be showed as $x_{h}=x_{h h}+x_{n h}$.

(1) Altitude fluctuation of flight track

$\theta_{p}$ is the pitch angle, and $V_{0}, \theta_{0}$ are the above. Then the flight altitude variation at $k$ point can be written as follows:

$$
x_{h h}=h(k)=V_{0} \sum_{j=1}^{k} \theta_{p}(j) \cdot \Delta t
$$

(2) Altitude fluctuation of pod relative to the airborne

When the helicopter flies smoothly, the pod inclination at the equilibrium position is I, and the small change of inclination is $\Delta I$. Taking pod swinging back for example, the altitude fluctuation caused by the pod can be expressed as follows:

$$
x_{n h}=L \cos \left(\frac{\pi}{2}-I\right)-L \cos \left(\frac{\pi}{2}-(I-\Delta I)\right)=\left(\cos \left(\frac{\pi}{2}-I\right)-\cos \left(\frac{\pi}{2}-I+\Delta I\right)\right) L=(\sin I-\sin (I-\Delta I)) L
$$

\section{Magnetic interference}

According to the distribution of the geomagnetic field, the magnetic interference is mainly caused by the latitude and altitude change of pod position. From Eq. (3) (7) (8) (9), the magnetic interference can be derived as follows:

$$
\Delta B=\Delta B_{l}+\Delta B_{h}=k_{l} \cdot\left(x_{h l}+x_{n l}\right)+k_{h} \cdot\left(x_{h h}+x_{n h}\right)
$$

Where $k_{l}$ and $k_{h}$ are the latitude gradient and altitude gradient of the geomagnetic field in measuring region.

In practical terms, the latitude fluctuation needs to calculate the north offset of the flight displacement and of the pod displacement relative to the aircraft, and the altitude fluctuation can be directly measured by radio altimeter and filtered.

\section{Simulation and analysis}

It is assumed that the flight speed $V_{0}=100 \mathrm{~m} / \mathrm{s}$, the sample frequency $f_{\mathrm{s}}=20 \mathrm{~Hz}$, the maneuvering angle $\Delta I_{m 1}=5^{\circ}$ corresponding to the center frequency, and maximum $8^{\circ}$ considering the random noise. The north offset of the pod displacement and the altitude fluctuation in eight courses are shown in 
Fig.3 and 4.

From Fig.3, it is known that the north offset is between $\pm 40 \mathrm{~m}$. There is just no north offset when the plane flies along the north-south direction. And along other directions, the north offset is obvious in the yaw stage. It can be seen from Fig. 4 that the altitude fluctuation is obvious in the pitch stage, and the range is about $\pm 60 \mathrm{~m}$.

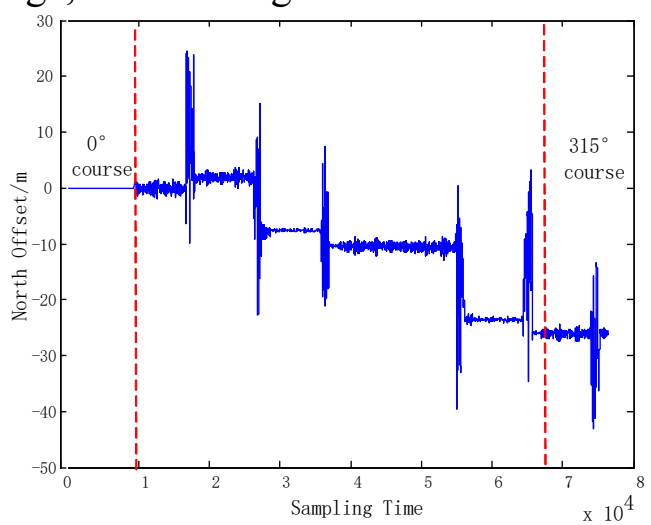

Fig.3 North offset of the pod displacement

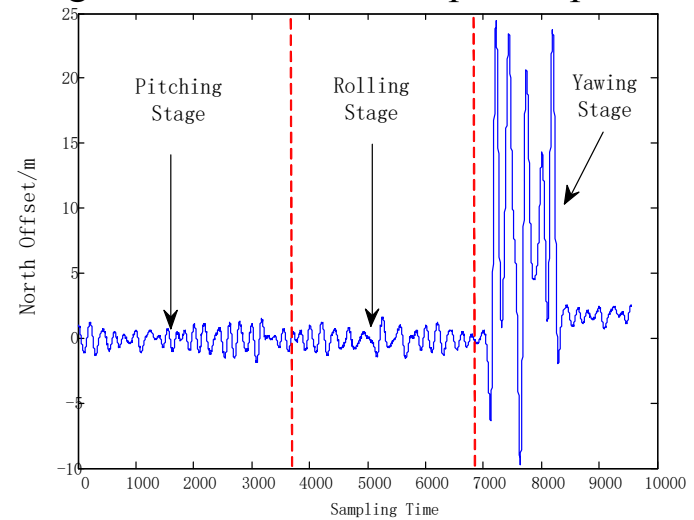

Fig.5 North offset of the pod displacement

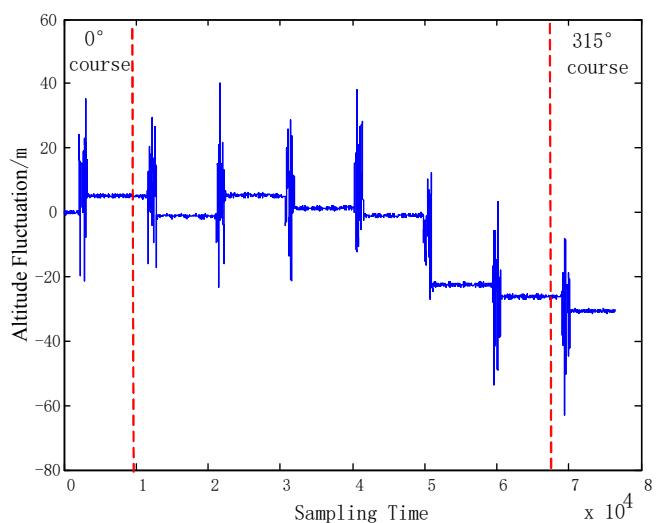

Fig.4 Altitude fluctuation of the pod

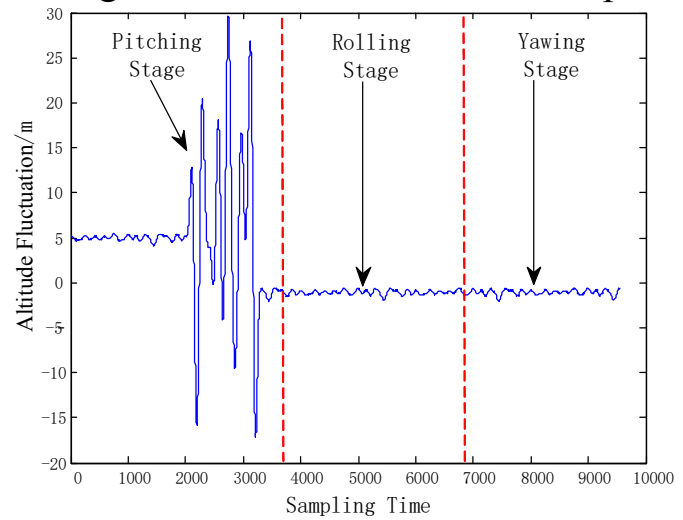

Fig.6 Altitude fluctuation of the pod

In order to clearly analyze the principles of the north offset and altitude fluctuation, we draw the curve in $45^{\circ}$ course for example, as shown in Fig.5 and 6.

If the magnetic field latitude gradient $k_{l}=5.91 \mathrm{nT} / \mathrm{km}$, altitude gradient $k_{h}=-25.91 \mathrm{nT} / \mathrm{km}$, we can obtain the magnetic interference according to the model, which showed in Fig.7.

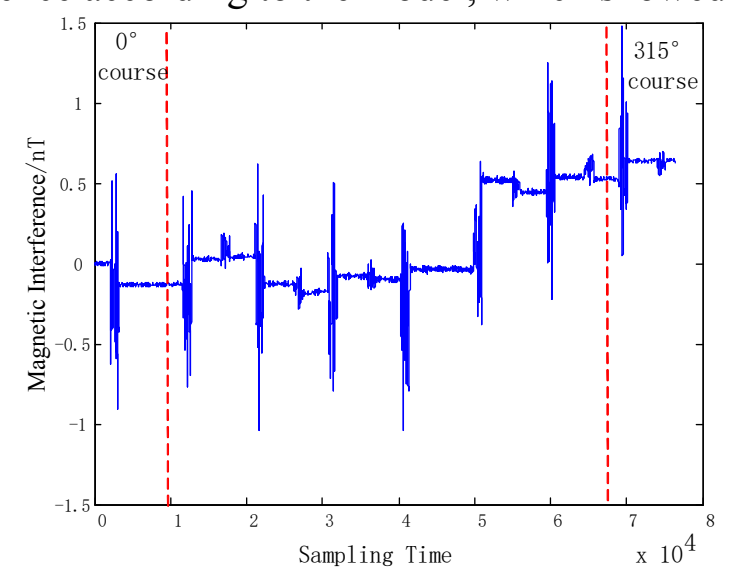

Fig. 7 Magnetic interference caused by pod fluctuation

From Fig 7, the magnetic interference caused by pod fluctuation is up to $\pm 1.0 \mathrm{nT}$, which is far greater than the resolution index of magnetic detector plane, so in order to detect magnetic target in long-distance and high-precision, we must compensate the magnetic interference.

\section{Conclusions}

Magnetic survey used in helicopter platform is different from fixed-wing aircraft and unmanned 
aerial vehicle. In order to find the principles, this paper has analyzed the background magnetic interference mechanism, simulated the small fluctuation, and set up calculation model. This work can be for the next step magnetic interference compensation, and provide a theoretical basis and data support for the flight test.

\section{Acknowledgements}

This paper is sponsored by National Natural Science Foundation of China (GN: 41374018).

\section{Reference}

[1] Li.BH. An Introduction of aeromagnetic survey system for light airplane.,Geophysical and Geochemical Exploration,18(1994) 228-229.

[2] Liu.XJ.Research on aeromagnetic compensation technology, Jilin University,2009

[3] Zhou.JJ. Research on Unmanned Aircraft's Magnetic Interference Compensation and Object Detection Method,Naval University of Engineering,2014.

[4] Bickel S H. Small signal compensation of magnetic fields resulting from aircraft maneuvers,IEEE Transactions on Aerospace and Electronic Systems, $15(1979)$ 518-525.

[5] Groom R W, Jia Ruizhong, Lo B. Magnetic compensation of magnetic noises related to aircraft's maneuvers in airborne survey, The Journal of Environmental and Engineering Geophysics, 9(2004) 101- 108.

[6] Zhou.JJ, Lin.CS, Chen.H. A method for aircraft magnetic interference compensation based on small signal model and LMS algorithm, Chinese Journal of Aeronautics, 27(2014)1578-1585.

[7] Qu XX, Li TL, Wang F. A study on the effect of bird's attitude on helicopter EM data and the correction method, Chinese Journal of Geophysics,57(2014)1310- 1321.

[8] Yin C C, Fraser D C. Attitude corrections of helicopter EM data using a superposed dipole model, Geophysics, 69(2004)431-439.

[9] Tan.B, Lin.CS, Huan.YC. Impacts of horizontal geomagnetic gradient on compensation precision of aircraft magnetic field, Journal of Naval University of Engineering, 24(2012) 75-79. 